\title{
Phase conjugation by four-wave mixing of statistical beams
}

\author{
G. Lera and M. Nieto-Vesperinas \\ Instituto de Optica "Daza de Valdés," Consejo Superior de Investigaciones Científicas, \\ Serrano 121, 28006 Madrid, Spain \\ (Received 11 September 1989)
}

\begin{abstract}
A study of the influence of the spatial structure and statistics of the pump beams in the phaseconjugation process is made, showing that the reflectivity varies along the phase-conjugation mirror when the phase factor in the coupling equations that describe the process is not constant. The mutual coherence function of the generated field is calculated from the mutual reflectivity of the mirror for two commonly used statistics for the partially coherent pump beams. Finally, the influence of diffractional effects is also analyzed, showing that, in general, the fidelity of the process is degraded.
\end{abstract}

\section{INTRODUCTION}

Phase conjugation by four-wave mixing (FWM) has been often studied by assuming the pump fields being counterpropagating plane waves. ${ }^{1,2}$ However, it would be desirable to have a theory that studies the case of spatially varying pump waves. This has been partially accomplished in Refs. 3-5, where TEM 00 pump beams and some generalizations have been considered. Also, an experiment with aberrated pump fields has been conducted confirming the quality of the conjugation when the pump waves are conjugates of each other. ${ }^{6}$

In this paper we attempt to expand further the theory of space-varying pump fields. We investigate the spatial coherence properties of the conjugation when depletion between the signal and the conjugated field occurs, but no diffractional effects are present, generalizing some results obtained in Ref. 5. Furthermore, we investigate the reflection characteristics when diffractional effects take place.

The problem we shall address is that concerning how the spatial structure of the beams and their coherence properties affect the process of phase conjugation (PC) by degenerate four-wave mixing (DFWM) in a Kerr-like medium. First, we shall consider no diffractional effects, later we shall study the influence of diffraction on the fidelity of the phase-conjugation mirror (PCM) reflectivity.

We assume that the two pumping beams (which from now on will be labeled 1 and 2), are much stronger than the signal and the "conjugated" beam. In this case, the nondepleted pump approximation ${ }^{7}$ is valid, and the equations that describe the coupling between the signal and the conjugated waves are

$$
\left(\Delta+k^{2}\right) \mathbf{E}_{s, c}=-\frac{4 \pi \omega^{2}}{c^{2}} \mathbf{P}_{s, c}
$$

where $k$ is the wave number and $\mathbf{E}_{s, c}$ are the electric fields for the signal and conjugated wave, according to whether the subscript is $s$ or $c$, respectively. $\omega$ is the frequency common to all the interacting beams (degenerate case).

The polarization function $\mathbf{P}_{s, c}$ is given by

$$
\mathbf{P}_{s, c}=\overleftrightarrow{\chi}: \mathbf{E}_{1} \mathbf{E}_{2} \mathbf{E}_{c, s}^{*}
$$

where $\overleftrightarrow{\chi}$ is the susceptibility tensor. We have in Eq. (2) a dyadic product. If all the beams are linearly polarized, we can take all these equations in terms of scalars corresponding to only one transversal component of the electric vectors with the only change of $\overleftrightarrow{\chi}$ by a $\chi_{0}$ (the effective susceptibility) which is given by the dyadic product of $\overleftrightarrow{\chi}$ with the polarization vectors of the different fields.

\section{PC WITHOUT DIFFRACTIONAL EFFECTS}

In this case, we may consider that our beams are described by

$$
\mathbf{E}=\widehat{\mathbf{o}} \boldsymbol{A}(\mathbf{r}) e^{i \mathbf{k} \cdot \mathbf{r}}
$$

where $\hat{\mathbf{o}}$ is de polarization vector of the field and $A(\mathbf{r})$ is slowly varying. By substituting Eq. (3) into (1), as it may be seen in standard texts on nonlinear optics ${ }^{7,8}$ we arrive at

$$
2 i \mathbf{k}_{s c,} \cdot \boldsymbol{\nabla} A_{s, c}=-\frac{4 \pi \omega^{2}}{c^{2}} P_{s, c} e^{-i \mathbf{k}_{s, c} \cdot \mathbf{r}} .
$$

In this case we can write for the left-hand side of Eq. (4)

$$
\mathbf{k}_{s, c} \cdot \nabla A_{s, c}=k\left(\cos \phi_{s, c}\right) \frac{\partial A_{s, c}}{\partial z}
$$

where $\phi_{s, c}$ is the angle between the $z$ axis and the wave vector $\mathbf{k}_{s, c}$. By making this substitution in Eq. (4) we obtain

$$
\begin{aligned}
& \frac{\partial A_{s, c}}{\partial z}=\frac{2 \pi i \omega^{2}}{c^{2} k \cos \phi_{s, c}} \chi_{0} A_{1} A_{2} A_{c, s}^{*} e^{i \Delta \mathbf{k} \cdot \mathbf{r}}, \\
& \Delta \mathbf{k}=\mathbf{k}_{1}+\mathbf{k}_{2}-\mathbf{k}_{c}-\mathbf{k}_{s},
\end{aligned}
$$

where $\Delta \mathbf{k}$ is the phase mismatch. In our calculations we assume $\Delta \mathbf{k}$ to be $\mathbf{0}$. In the case of PC this means that if we take $\mathbf{k}_{2}=-\mathbf{k}_{1}$ the wave vector of the signal and conjugated beams will satisfy the same relation: $\mathbf{k}_{s}=-\mathbf{k}_{c}$. In such a case we have that $\phi_{c}=\phi_{s}+\pi$.

With all these changes, Eqs. (5) are transformed into 


$$
\begin{aligned}
& \frac{\partial A_{s}^{*}}{\partial z}=H^{*}(\mathbf{r}) A_{c}(\mathbf{r}), \\
& \frac{\partial A_{c}}{\partial z}=-H(\mathbf{r}) A_{s}^{*}(\mathbf{r}),
\end{aligned}
$$

$H(\mathbf{r})$ being

$$
\begin{aligned}
& H(\mathbf{r})=\frac{2 \pi i \omega^{2}}{c^{2} k \cos \phi_{s}} \chi_{0} A_{1} A_{2}=i K(\mathbf{r}) e^{i \Phi(\mathbf{r})}, \\
& K(\mathbf{r})=|H(\mathbf{r})| .
\end{aligned}
$$

Once one has arrived at this point, several approximations may be made in order to find an analytical solution to Eqs. (6). The simpler case of $\Phi(r)=0$ has been studied in Ref. 4. This condition is equivalent to Eq. (11) in this reference.

We follow a method similar to that given in Ref. 9 and use the case where $\Phi(r)$ is given by the following form:

$$
\Phi(\mathbf{r})=2 B(\mathbf{r}) q+\Phi(\mathbf{R}, 0)
$$

where $B$ and $\Phi$ are arbitrary functions of $\mathbf{R}, \mathbf{r}=(\mathbf{R}, z)$, and $q$ is given by

$$
q(\mathbf{R}, z)=\int_{0}^{z} K(\mathbf{r}) d z
$$

We can then obtain the value of the conjugated field in the plane $z=0$ of the PCM:

$$
E_{c}(\mathbf{R}, 0)=\frac{i(\tan \Omega) e^{i \Phi(\mathbf{R}, 0)}}{\Omega / Q-i B(\mathbf{R}) \tan \Omega} E_{s}^{*}(\mathbf{R}, 0)
$$

where $Q(\mathbf{R})=q(\mathbf{R}, L), \quad \Omega(\mathbf{R})=\left\{1+[B(\mathbf{R})]^{2}\right\}^{1 / 2} Q(\mathbf{R})$, and $L$ is the crystal length.

The main differences between this result and the standard one ${ }^{1}$ are that here we have a dependence in the ratio between the conjugate field and the signal with the point of the surface of the PCM. Thus, different parts of the signal transversal profile are "reflected" in different ways depending on the place of the PCM where they arrive. Moreover, there is a phase factor that affects the fidelity of the conjugation process.

To analyze the statistical properties of the conjugated field we consider the mutual coherence function of a field which is defined as

$$
\Gamma\left(\mathbf{R}_{1}, \mathbf{R}_{2}\right)=\left\langle E\left(\mathbf{R}_{1}\right) \mathbf{E}^{*}\left(\mathbf{R}_{2}\right)\right\rangle .
$$

One of the most important characteristics of a mirror is its reflectivity. In the case of a PCM we shall consider in what follows the mutual reflectivity which we define as

$$
G\left(\mathbf{R}_{1}, \mathbf{R}_{2}\right)=\frac{\Gamma_{c}\left(\mathbf{R}_{1}, \mathbf{R}_{2}\right)}{\Gamma_{s}^{*}\left(\mathbf{R}_{1}, \mathbf{R}_{2}\right)}
$$

When the two arguments of $G$ are the same, say $\mathbf{R}$, we obtain the reflectivity $(\mathcal{R})$ at $\mathbf{R}$. Now we are going to analyze how the different properties of the pumping and signal beams affect the mutual reflectivity $G$.

From Eqs. (7), (8), and (9) it can be seen that a sufficient condition to obtain a PCM whose response (which is characterized by $G$ ) is independent of the signal (this being desirable in order to be capable of analyzing the gen- eral properties of the PCM) is that the signal has to be statistically independent of the pumping beams. In this case the mutual reflectivity according to Eqs. (7), (8), and (9) is given by

$G\left(\mathbf{R}_{1}, \mathbf{R}_{2}\right)=\left\langle\frac{\left(\tan \Omega_{1}\right)\left(\tan \Omega_{2}\right) e^{i\left[\Phi\left(\mathbf{R}_{1}, 0\right)-\Phi\left(\mathbf{R}_{2}, 0\right)\right]}}{\left[\Omega_{1} / Q_{1}-i B_{1} \tan \Omega_{1}\right]\left[\Omega_{2} / Q_{2}+i B_{2} \tan \Omega_{2}\right]}\right\rangle$

where $\Omega_{1}$ and $Q_{2}$ stand for $\Omega\left(\mathbf{R}_{1}\right)$ and $Q\left(\mathbf{R}_{2}\right)$, respectively.

\section{INFLUENCE OF THE SPATIAL STRUCTURE OF THE PUMP BEAMS}

In this section we shall suppose that we have pumping waves with Gaussian transversal profile but without statistical fluctuations:

$$
E_{j}(\mathbf{r})=A_{j} e^{-\left[k_{j}^{2} r^{2}-\left(\mathbf{k}_{j} \cdot \mathbf{r}\right)^{2}\right] /\left(k_{j}^{2} W^{2}\right)} e^{i \mathbf{k}_{j} \cdot \mathbf{r}} \quad(j=1,2) .
$$

$W$ is the spot size and we take $\mathbf{k}_{1}+\mathbf{k}_{2}=\mathbf{0}$. With this kind of profile we obtain for the mutual reflectivity, given by Eq. (10) (assuming $B(\mathbf{R})=0$ ),

$$
G\left(\mathbf{R}_{1}, \mathbf{R}_{2}\right)=\tan \left(Q_{1}\right) \tan \left(Q_{2}\right) .
$$

In Fig. 1 we show the reflectivity $[R(R)=G(R, R)]$ for different directions of propagation of the pump waves. If we take $\mathbf{k}_{1}$ in the $x z$ plane, it is expressed as

$$
\mathbf{k}_{1}=k(\sin \theta, 0, \cos \theta) \text {. }
$$

The calculations of Fig. 1 have been done under the following conditions. $K(0)=1 / L$ and $L=W$, for four different values of $\theta$. It can be seen that the reflectivity does not depend on $\mathbf{R}$ for $\theta=\pi / 2$ only. This particular case corresponds to the main result of Ref. 9, namely, in

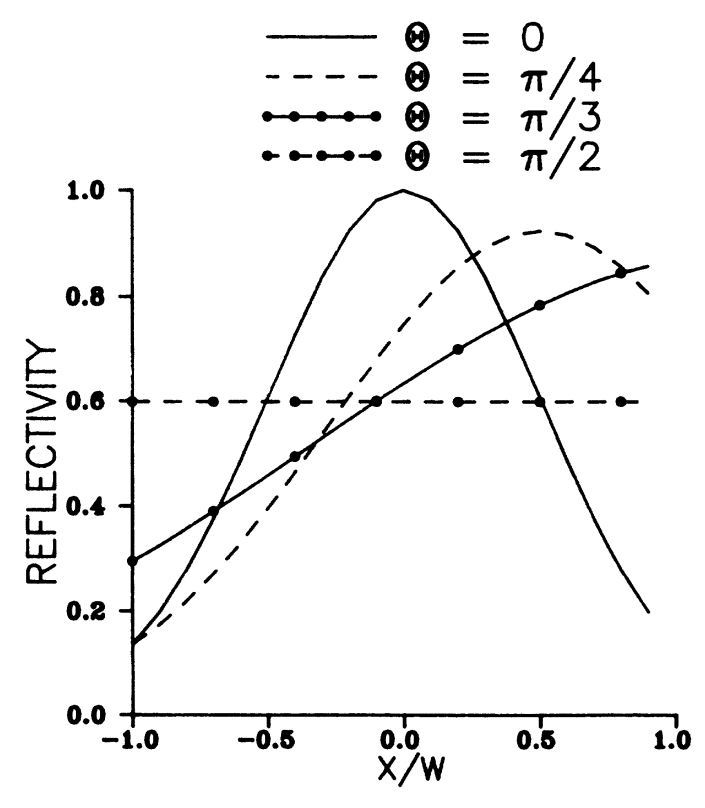

FIG. 1. Reflectivity $[R(R)]$ for four different directions of propagation of the pump waves. $\theta=0, \pi / 4, \pi / 3, \pi / 2$. 
this special case, the influence of the finite profile only affects the interaction length, but not the features of the output beam. This is, of course, due to the fact that we consider (as in Ref. 9) that the spatial distribution of the pump beams does not vary with the propagation; that is, there are no diffractional effects, and also we disregard the possible appearance of other nonlinear effects, like self-focusing, that may vary this spatial distribution.

\section{PUMP FIELDS WITH STATISTICS DUE TO A RANDOM-PHASE SCREEN}

Once the effect of a Gaussian profile for the pump beams has been studied, we can use this result to analyze the effect of only the partial coherence of the pumps. This is a priori interesting because it is well known that nonlinear optical processes are significantly influenced by the coherence properties of the sources, and moreover, it has been proven in other works ${ }^{10-12}$ that in these processes the efficiency can be increased significantly by the partial coherence of the input beams. In this kind of studies several particular statistics are generally used. ${ }^{10-12}$

We shall assume, first, that the randomness in the pumping fields occurs only in their phases, as is the case of beams passing through a random-phase screen. ${ }^{10}$

We shall employ a colineal geometry. This geometry has been used in several experimental setups to study the angular dependence of PC in other kinds of media, like $\mathrm{SF}_{6}{ }^{13}$ In this case we consider the pumps as being described by

$$
E_{j}(\mathbf{r})=A_{j} e^{i \phi(\mathbf{R})} e^{i \mathbf{k} \cdot \mathbf{r}} e^{-R^{2} / W^{2}}, \quad j=1,2
$$

where $\phi$ is a random phase whose statistics fulfills

$$
\begin{aligned}
& \langle\phi(\mathbf{R})\rangle=0 \quad \forall \mathbf{R}, \\
& \left\langle\phi\left(\mathbf{R}_{1}\right) \phi\left(\mathbf{R}_{2}\right)\right\rangle=\sigma^{2} \exp \left(-\frac{\left(\mathbf{R}_{1}-\mathbf{R}_{2}\right)^{2}}{\xi^{2}}\right) \forall \mathbf{R}_{1}, \mathbf{R}_{2},
\end{aligned}
$$

$\xi$ being the coherence length and $\sigma$ being the root mean square of $\phi$.

In order to separate the effect of the spatial structure of the beams we have studied the ratio between the mutual reflectivity in this case of partial coherence and in the former case of complete coherence for the value $\theta=0$. This will be called the normalized mutual reflectivity $(\bar{G})$.

With the only assumption that $\phi\left(\mathbf{R}_{1}\right)$ and $\phi\left(\mathbf{R}_{2}\right)$ are two jointly distributed Gaussian random variables ${ }^{14}$ it can be easily shown that [again $B(\mathbf{R})=0$ ]

$$
\bar{G}\left(\mathbf{R}_{1}, \mathbf{R}_{2}\right)=\exp \left\{-2 \sigma^{2}\left[1-\exp \left(-\frac{\left(\mathbf{R}_{1}-\mathbf{R}_{2}\right)^{2}}{\xi^{2}}\right]\right)\right\} .
$$

In Fig. $2 \bar{G}$ is represented as a function of $\left|\mathbf{R}_{1}-\mathbf{R}_{2}\right|$ in spot size units, for four different values of the ratio $\xi / W$. We can see that $\bar{G}$ always decreases with the separation between the points of the surface of the mirror, but an interesting feature is that in this kind of statistics, even for greater values of $\xi$, it does not go to zero, approaching an asymptotic value $\exp \left(-2 \sigma^{2}\right)$. In the case shown in Fig. 2 , we have taken $\sigma=1$.

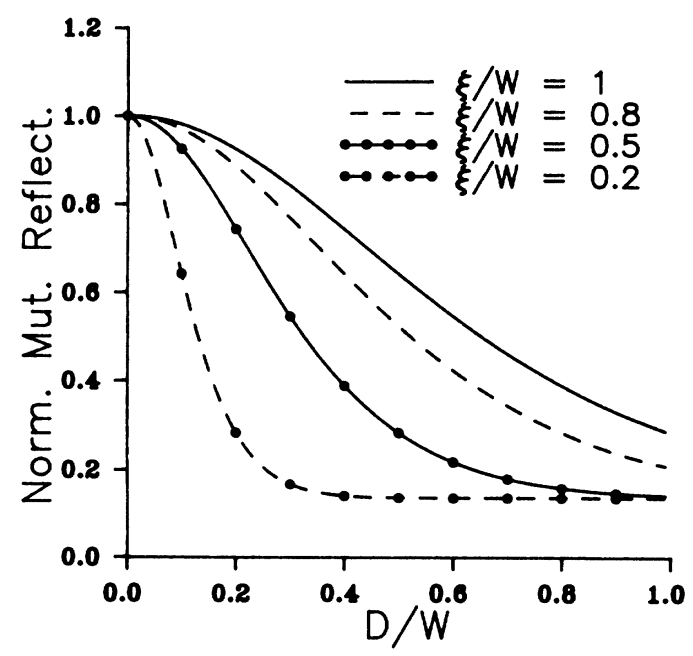

FIG. 2. Normalized mutual reflectivity $(\bar{G})$ as a function of $\left|\left(\mathbf{R}_{1}-\mathbf{R}_{2}\right) / W\right|$, for four values of $\xi / W$ (random-phase screen statistics).

\section{PUMP FIELDS WITH GAUSSIAN STATISTICS}

In this section we are going to assume that the real and imaginary parts of each pump field are independent Gaussian variables, and that the real (imaginary) part of the field at different points of the profile are Gaussian variables with Gaussian correlation. This kind of statistics has been found to accurately model the behavior of a partially coherent light beam ${ }^{15}$ and also of beams generated by a "Gaussian Schell source." "11,16 An additional supposition that we made here is that $Q(\mathbf{R})<<1$ in order to simplify the calculations. We have found two interesting limiting cases.

In the first one, we take the pumping fields as being completely independent of each other. Straightforward calculations lead to

$$
\bar{G}\left(\mathbf{R}_{1}, \mathbf{R}_{2}\right)=e^{-2\left(\mathbf{R}_{1}-\mathbf{R}_{2}\right)^{2} / \xi^{2}}
$$

In Fig. 3 we show $\bar{G}$ against the distance between the

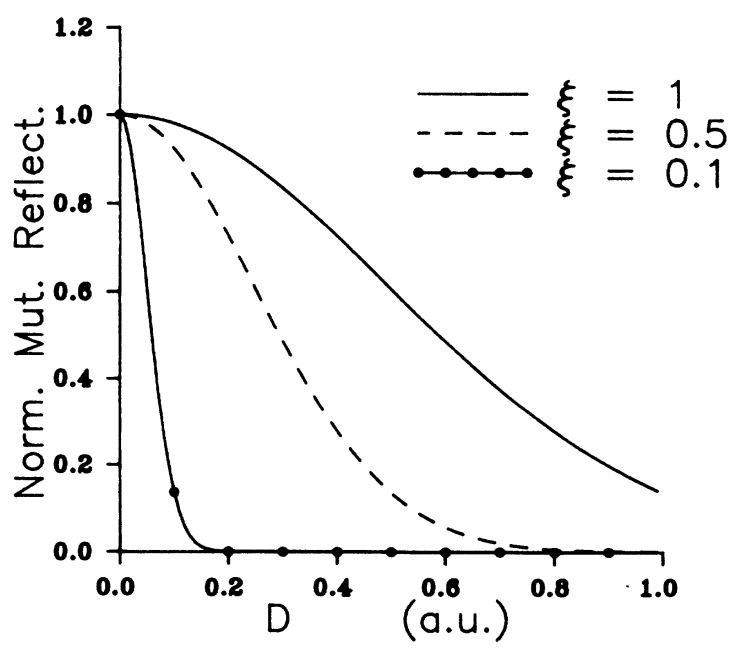

FIG. 3. Normalized mutual reflectivity $(\bar{G})$ as a function of $\left|\mathbf{R}_{1}-\mathbf{R}_{2}\right|$, for different values of $\xi$ (Gaussian statistics). 
points of the surface of the PCM under consideration, for different values of the coherence radius $\xi$. It can be compared with Fig. 2 and we see that the general behavior is the same, decreasing $\bar{G}$ with the distance, but in this case $\bar{G}$ tends to zero, while in the other case it does not. From Eq. (11) it is easy to see that the normalized reflectivity $\bar{R}(\mathbf{R})=\bar{G}(\mathbf{R}, \mathbf{R})$ has always a value equal to 1 .

In the second case we have taken the pumping fields as being completely dependent of each other, as is in many experimental setups where there is only one pump beam which is reflected in the backside of the nonlinear medium, and the reflected beam plays now the role of the second pump field. It is easy to calculate directly the $\bar{R}(\mathbf{R})$, because what we have is that, due to the approximations adopted, the only random variable that remains in $\bar{R}$ is the intensity of the pump beam, which follows a negative exponential distribution

$$
P(I)=\frac{1}{\langle I\rangle} e^{-I /\langle I\rangle} .
$$

By calculating the expected value of Eq. (10) with this statistics one arrives at the result of $\bar{R}(\mathbf{R})=2$. Here we can see the reason to use a partially coherent pump beam: Like in the case of second-harmonic generation ${ }^{10-12}$ (SHG) we have found that the efficiency of the process (in this case the reflectivity) may be enhanced by the use of partially coherent beams. However in SHG the purpose is only to obtain a SHG beam, the more intense the better. In PC our main goal is not only to obtain a very intense response, but also a good quality one, and for this it is not enough that the $\overline{\mathcal{R}}$ does not depend on $\mathbf{R}$; it is also necessary that $G$ be independent of the position of the PCM. If we are able to obtain a great coherence radius $\xi$ we can still take the advantage of having a greater response.

\section{PC WITH DIFFRACTIONAL EFFECTS}

In this section we are going to study how the DFWM process is affected by the finite extension of the beams. The couple beam formalism leads us to a set of equations which describe the propagation of the signal and conjugate beams inside the nonlinear medium under the paraxial approximation. We assume that both beams are propagating with their axis close to the $z$ axis. The basic equations for this case are

$$
\begin{aligned}
& \hat{D}^{+} A_{s}=-\frac{4 \pi \omega^{2}}{c^{2}} e^{-i k z} P_{s}(\mathbf{r}), \\
& \hat{D}^{-} A_{c}=-\frac{4 \pi \omega^{2}}{c^{2}} e^{i k z} P_{c}(\mathbf{r}),
\end{aligned}
$$

where $A_{s, c}$ are the slowly varying part of the fields, and

$$
\begin{aligned}
& E_{s}(\mathbf{r})=A_{s} e^{i k z}, \quad E_{c}(\mathbf{r})=A_{c} e^{-i k z}, \\
& \hat{D}^{ \pm}=\frac{\partial^{2}}{\partial x^{2}}+\frac{\partial^{2}}{\partial y^{2}} \pm 2 i k \frac{\partial}{\partial z}
\end{aligned}
$$

If we try to find from this set a new equation in which only one of the fields is involved, we find a fourth-order linear differential equation, which in the case of plane pump waves is simplified to

$$
\begin{aligned}
& \begin{array}{l}
\chi(\mathbf{r})=\chi_{0} E_{1}(\mathbf{r}) E_{2}(\mathbf{r})=\chi_{0} E^{2} e^{i(\mathbf{a} \cdot \mathbf{R}+b z)}, \\
\hat{D}^{-} \hat{D}^{-} A_{c}= \\
\quad\left(2 b k+a^{2}\right) \hat{D}^{-} A_{c}+2 i \mathbf{a} \cdot \boldsymbol{\nabla}\left(\hat{D}^{-} A_{c}\right) \\
\quad+4 k^{2}|\boldsymbol{M}|^{2},
\end{array} \\
& \begin{array}{c}
M=\frac{2 \pi i \omega^{2} \chi_{0} E^{2}}{k c^{2}} .
\end{array}
\end{aligned}
$$

The solution of this equation is easily found to be, under the usual conditions for $\mathrm{PC}\left[E_{c}(\mathbf{R}, L)=0\right]$,

$$
\begin{aligned}
E_{c}(\mathbf{R}, 0)= & i \tan (|M L|)\left(1+i \frac{2 b k+a^{2}}{4 k} \frac{\tan (|M L|)}{|M|}\right) \\
& \times E_{s}^{*}(\mathbf{R}, 0)-i \frac{\tan ^{2}(|M L|)}{|M|} \frac{\mathbf{a} \cdot \nabla E_{s}^{*}(\mathbf{R}, 0)}{2 k},
\end{aligned}
$$

with the only supposition that

$$
\frac{b^{2}}{k^{2}}, \frac{a^{4}}{k^{4}}<<1, \quad \Delta \mathbf{k}=(\mathbf{a}, b)
$$

It can be seen from Eq. (13), that if $\Delta \mathbf{k}=\mathbf{0}$ the spatial structure does not influence the features of the PC (at least under the paraxial approximation and with plane pumping waves), because we obtain the classical result of Ref. 1. However, the appearance of the last term of Eq. (13) changes completely the result of the process, because this term is not proportional to the conjugate of the signal field, but to its gradient. From Eq. (13) it is possible to find under what condition a good conjugate is to be expected.

There are mechanisms, however, which may lead to a phase mismatch, as can be that the pumping waves are not exactly counterpropagating, or if their frequencies do not coincide with one another or with the frequency of the signal. A brief discussion of other sources for this mismatch can be found in Ref. 17. To carry on our analysis, we may suppose that the phase mismatch is due to a change of the propagation direction of one of the pump beams. Since, however, the results do not depend on the physical origin of the mismatch, they will be valid when the value of the mismatch is similar to the one used here, even though due to a different cause.

We take one of the beams, say $E_{1}$, propagating exactly in the $x$-axis direction, and the other one almost counterpropagating, but with a little component in the $y$-axis direction. By taking the paraxial approximation, we can say that the phase mismatch is given by

$$
\Delta \mathbf{k}=\left(\frac{k_{y}^{2}}{2 k}, k_{y}, 0\right)=\mathbf{k}_{1}+\mathbf{k}_{2} .
$$

In order to simplify, we take the signal field at the plane $z=0$ as being independent of $x$ and real. If we define $\bar{R}$ as the ratio between the reflectivity obtained in this case and in the case $\Delta \mathbf{k}=\mathbf{0}$, we obtain 


$$
\begin{aligned}
\bar{R}(\mathbf{R})= & {\left[1-\frac{\tan (|M L|)}{|M|} \frac{k_{y}}{2 k} \frac{\partial \ln A_{s}}{\partial y}\right)^{2} } \\
& +\frac{\tan ^{2}(|M L|)}{|M|^{2}} \frac{k_{y}^{4}}{16 k^{2}} .
\end{aligned}
$$

We are going to assume that the mismatch is not very large, so that the last term in Eq. (14) can be neglected.

Our aim is to analyze now how the PC process is affected by several parameters that define the spatial distribution of a beam with Gaussian spatial structure but with a given modulation. As an example, we shall consider a sinusoidal modulation, in the case in which we have a nonzero phase mismatch. Hence we take the distribution of the signal field in the $z=0$ plane as being

$$
A_{s}(\mathbf{R}, 0)=e^{-\left(y^{2} / W^{2}\right)} \sin (h y+\alpha) \text {. }
$$

We have done calculations for the normalized reflectivity for several values of $h, W$, and the interaction length. We define two new parameters:

$$
p=\frac{\tan (|M L|)}{|M|} \frac{k_{y}}{2 k} h, \quad q=\frac{p}{h W} .
$$

In Fig. $4 \overline{\mathscr{R}}$ is shown for the spot size equal to $20 \pi / h$, and for different values of $p$. This can be achieved by varying the interaction length. For the smallest value of $p \overline{\mathcal{R}}$ tends quickly to one after decaying to zero when $h y$ increases. This means that in that case, the only differences with the case of perfect matching are at those points in which the signal is zero. Therefore the only thing that takes place in this case is a reduction in the visibility. If we increase the value of $p$ the shape of $\bar{R}$ changes, being more different with respect to the matched case. The positions of the zeros are changed, too. For $p=0.3$ the "conjugated" is quite distorted, as manifested by the poles of the $\bar{R}$ curve versus $h y$.

In Fig. 5 we show $\bar{R}$ versus $h y$ for different values of the spot size $W$ for $\alpha=0.02$ and $p=0.05$. The value 1 of

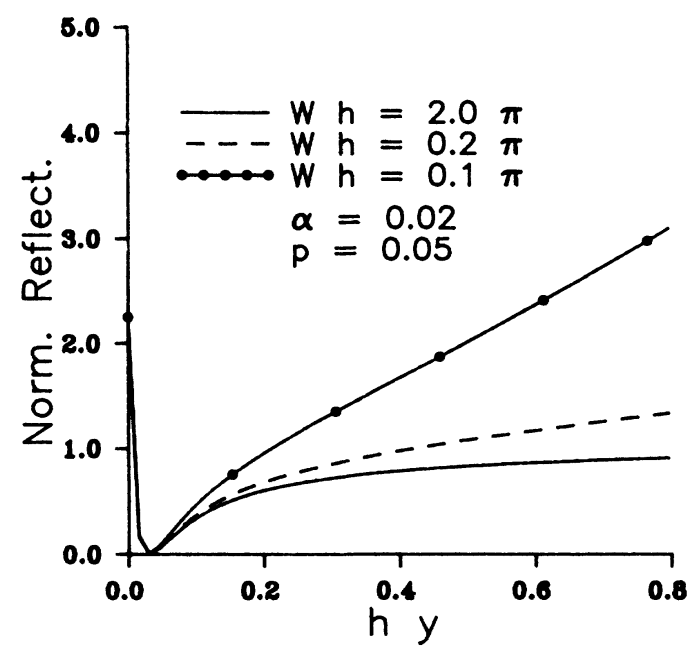

FIG. 5. Normalized reflectivity $(\overline{\mathcal{R}})$ along the PCM's surface (hy) for $p=0.05$, and different values of the spot size.

$\bar{R}$ corresponds to the matched case. We can see that there is a strong dependence of this function with the spot size; that is, with the finite extension of the beam. Naturally, the results for $\bar{R}$ are only significant for $y \leq 2 W$ approximately, because for values of $y$ greater than $2 W$ the intensity of the signal field is practically zero.

Even for very low values of $p$ there are points where $\bar{R}$ goes to infinity (those points where the signal field is zero). This means that for these points the output is not zero, the visibility of the output image being degraded. On the other hand, we can see that there are points at which $\bar{R}$ is zero, so that, the fidelity of the conjugation at these points is lost once again.

In Fig. 6 we show $\bar{R}$ for $p=0.3$ and the same values of the other parameters. Because of the greater value of $p$ this time, the changes in $\bar{R}$ are very great and we find

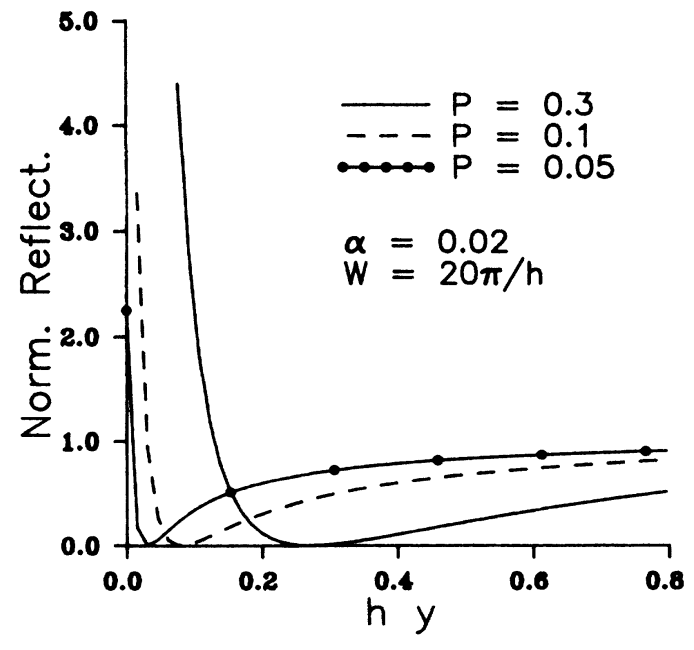

FIG. 4. Normalized reflectivity $(\bar{R})$ along the PCM's surface (hy) for different values of $p$.

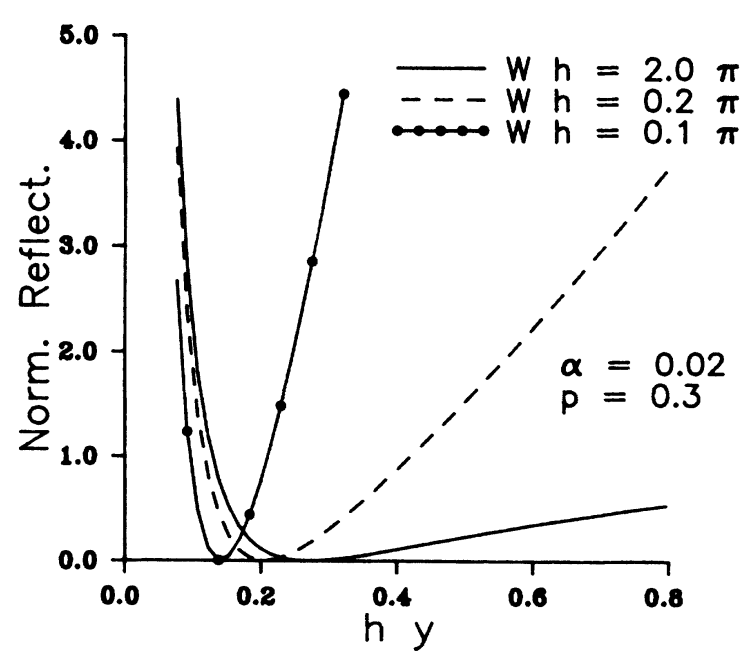

FIG. 6. Normalized reflectivity $(\overline{\mathcal{R}})$ along the PCM's surface ( hy) for $p=0.3$, and different values of the spot size. 


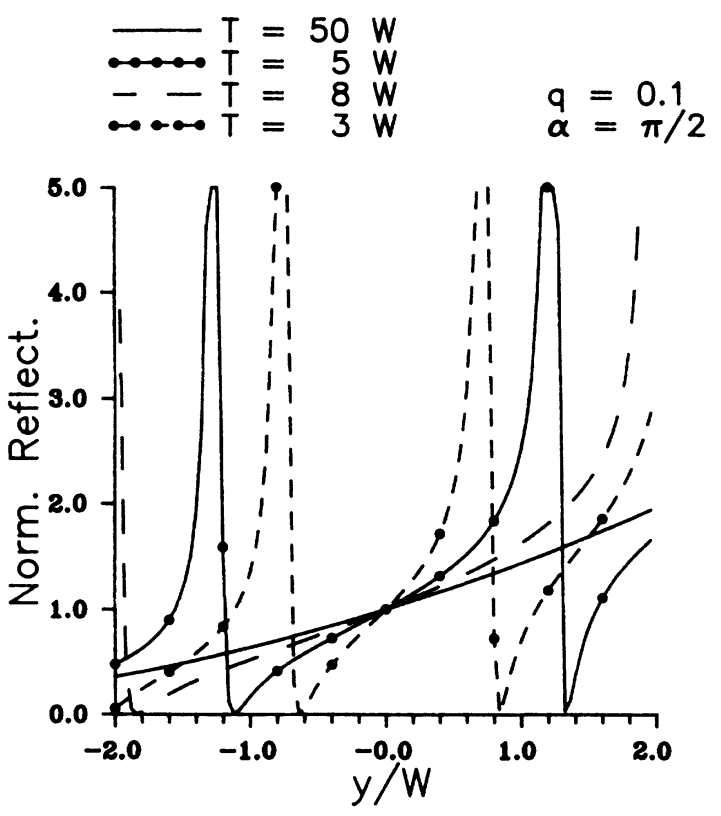

FIG. 7. Normalized reflectivity $(\overline{\mathcal{R}})$ along the PCM's surface $(y / W)$ with $W$ fixed and different values of the spatial frequency.

that here the "conjugation" process really does not work. Another different feature that can be observed here is that the zeros of $\bar{R}$ are placed at different points for different values of $W$.

In Fig. 7 we represent $\bar{R}$ for the same value of $W$ and vary the value of the spatial frequency $h$. The corresponding values of the spatial period are $T=50 \mathrm{~W}, 8 \mathrm{~W}$, $5 W$, and $3 W$. Here we have taken $\alpha$ to be $\pi / 2$ and $q=0.1$. Only when $T / W$ is very large one can expect the image to be slightly distorted. In all other cases the output image presents a very different behavior than that of the input.

From these figures, we can conclude, that the reflectivity depends strongly on the spatial structure of the fields, if there is a phase mismatch between the pumping beams. On the other hand, we know that there are many factors ${ }^{17}$ that may produce the appearance of this mismatch. Even if the mismatch is very small, there is a

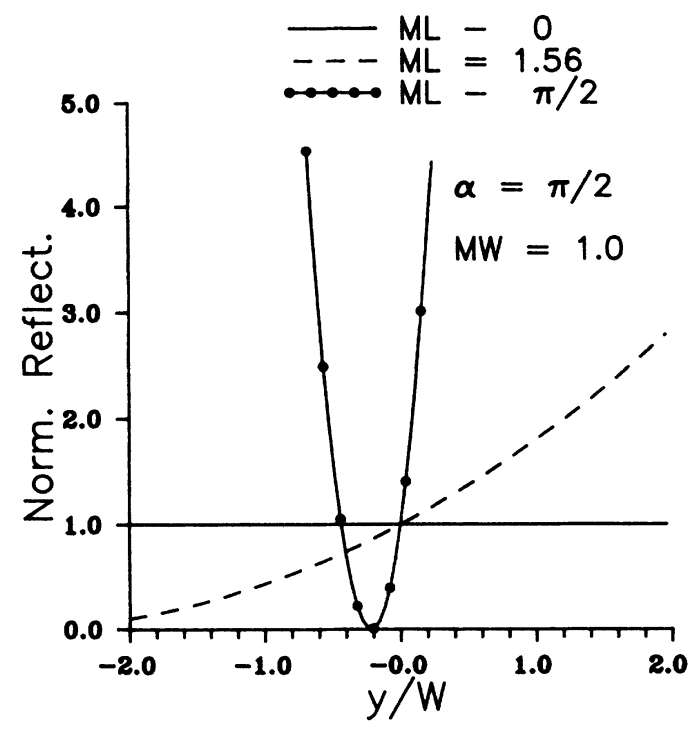

FIG. 8. Normalized reflectivity $(\overline{\mathcal{R}})$ along the PCM's surface $(y / W)$ for different values of the interaction length $L$.

loss in the visibility due to the fact that the reflectivity tends to infinity in those points in which the signal is null. If the mismatch is not so small, the fidelity in the conjugation process is being gradually lost. For a Gaussian beam of the kind studied here a natural condition to have a good quality conjugation is that $p, q<<1$.

In Fig. 8 we analyze how $\bar{R}$ varies for a given value of the phase mismatch $\left(k_{y} / k=0.002\right)$, for different values of the interaction length. Here we take $|M W|=1$ and $\alpha=\pi / 2$. For this small value of the phase mismatch, we can see the behavior of the instability in the response when one goes to the regime of oscillation $|M L| \rightarrow \pi / 2$.

\section{ACKNOWLEDGMENTS}

This work was supported by the Comisión Interministerial de Ciencia y Tecnología under Grant No. PB 0278. G. Lera acknowledges a grant from the Ministerio de Educacion y Ciencia.
${ }^{1}$ A. Yariv and D. M. Pepper, Opt. Lett. 1, 16 (1977).

${ }^{2}$ A. Yariv, IEEE J. Quantum Electron. QE-14, 650 (1978).

${ }^{3}$ R. Trebino and A. Siegman, Opt. Commun. 32, 1 (1980).

${ }^{4}$ E. Bochove, J. Opt. Soc. Am. 73, 1330 (1983).

${ }^{5}$ B. Crosignani and A. Yariv, J. Opt. Soc. Am. A 1, 1034 (1984).

${ }^{6}$ M. D. Skeldon, P. Narum, and R. W. Boyd, Opt. Lett. 12, 343 (1987).

${ }^{7}$ Y. R. Shen, Principles of Non Linear Optics (Wiley, New York, 1984).

${ }^{8}$ N. Bloembergen, Non-Linear Optics (Benjamin, New York, 1965).

${ }^{9}$ J. W. Haus, C. M. Bowden, and C. C. Sung, Phys. Rev. A 35, 3398 (1987).
${ }^{10}$ M. S. Zubairy and J. K. McIver, Phys. Rev. A 31, 856 (1985).

${ }^{11}$ N. A. Ansary and M. S. Zubairy, Opt. Commun. 59, 385 (1986).

${ }^{12}$ M. Nieto-Vesperinas and G. Lera, Opt. Commun. 69 (1989).

${ }^{13}$ C. L. Cesar et al., Opt. Lett. 13, 12 (1988).

${ }^{14} \mathrm{~J}$. W. Goodman, Statistical Optics (Wiley, New York, 1985).

${ }^{15}$ V. A. Aleshkevich, S. S. Lebedev, and A. N. Matveev, Zh. Eksp. Teor. Fiz. 83, 1249 (1982) [Sov. Phys._JETP 56, 715 (1982)].

${ }^{16}$ G. Lera and M. Nieto-Vesperinas, Opt. Commun. 73, 244 (1989).

${ }^{17}$ B. Ya. Zel'dovich, N. F. Pilipetsky, and V. V. Shkunov, Principles of Phase Conjugation (Springer-Verlag, Berlin, 1985). 